\title{
INTEGRALS OF CONFLUENT HYPERGEOMETRIC FUNCTIONS
}

\author{
GIOVANNA PITTALUGA
}

\begin{abstract}
A.bstract. The moments of the weight functions $w(x)=e^{-x} x^{\mu}(\ln x)^{\rho}, \rho=0,1,2$, on $[0, \infty)$ with respect to the Confluent Hypergeometric function $\phi(a-n, c ; x), n=$ $0,1,2, \ldots$, are explicitly evaluated.
\end{abstract}

\section{Introduction}

In a previous paper Blue [1] gave a simply expression for the integral $\int_{0}^{1} p_{n}(2 x-1) \ln (1 / x) d x$, where $P_{n}(2 x-1)$ is the shifted Legendre polynomial. Gautschi [5] treated $\int_{0}^{1} x^{\alpha} \ln (1 / x) P_{n}(2 x-1) d x, \alpha>-1$.

The evaluation of these integrals is related to the construction of the modified moments with respect to certain classes of polynomials [4].

Afterwards Gatteschi [2] generalized these results by considering and explicitly evaluating the modified moments of the weight functions $w(x)=x^{\rho}(1-x)^{\alpha} \ln (1 / x), \alpha, \rho>-1$, on $[0,1]$, with respect to the shifted Jacobi polinomials $P_{n}^{*(\alpha, \beta)}(x)=P_{n}^{(\alpha, \beta)}(2 x-1)$, and $w_{p}(x)=x^{\rho} e^{-x}(\ln x)^{p}, \rho>-1, p=1,2$ on $[0, \infty)$, with respect to the generalized Laguerre polynomials $L_{n}^{(\alpha)}(x)$.

In a more recent paper [6], Kalla, Conde and Luke have considered a different problem, not necessarily related to the modified moments. More precisely, they have examined the integral $\int_{-1}^{1}(1-x)^{a}(1+x)^{b} P_{\nu}^{(\alpha, \beta)}(x) d x, \operatorname{Re}(a), \operatorname{Re}(b)>-1$, and its partial derivatives with respect to $a$ and $b$, where $P_{\nu}^{(\alpha, \beta)}(x)$ is the Jacobi function which reduces to the Jacobi polynomial if $\nu$ is a positive integer. Gatteschi's result about Laguerre polynomial integrals has been considered again by Kalla and Conde in a more recent paper [7].

The purpose of this paper is to evaluate the moments of the weight functions $w(x)=$ $e^{-x} x^{\mu}(\ln x)^{\rho}, \rho=0,1,2, \ldots$ on $[0, \infty)$ with respect to the Confluent Hypergeometric function $\phi(a, c ; x)$, that is the integrals

$$
I_{\rho}(a, \mu)=\int_{0}^{\infty} e^{-x} x^{\mu}(\ln x)^{\rho} \phi(a, c ; x) d x, c \neq 0,-1,-2, \ldots, \mu>-1, \rho=1,2, \ldots
$$

Received February 17, 1989.

1980 Mathematics Subject Classification. Primary 33A30.

This work was supported by the Consiglio Nazionale delle Ricerche and by the Ministero della Pubblica Istruzione. 
Since, by putting

$$
J(a, \mu)=\int_{0}^{\infty} e^{-w} x^{\mu} \phi(a, c ; x) d x, \quad c \neq 0,-1,-2, \ldots, \quad \mu>-1,
$$

it is

$$
I_{\rho}(a, \mu)=\frac{\partial^{\rho}}{\partial \mu^{\rho}} J(a, \mu), \quad \rho=1,2, \ldots
$$

the evaluation of integrals (1.1) follows from that of $J(a, \mu)$ and its partial derivatives with respect to $\mu$.

Here and throughout this paper, we use the notation of Gatteschi [3].

\section{Thtegral- $J(a, \mu)$ and related derivatives}

For the computation of the integral $J(a, \mu)$ we make use of the known expansion [3, p.61]

$$
\phi(a, c ; x)=\sum_{k=0}^{\infty} \frac{(a)_{k}}{(c)_{k}} \frac{x^{k}}{k !} .
$$

Indeed, by using (2.1) and termwise integration, we can write

$$
\begin{aligned}
J(a, \mu) & =\Gamma(\mu+1) \sum_{k=0}^{\infty} \frac{(a)_{k}(\mu+1)_{k}}{(c)_{k}} \frac{1}{k !} \\
& =\Gamma(\mu+1) F(a, \mu+1 ; c ; 1),
\end{aligned}
$$

where $F(a, \mu+1 ; c ; 1)$ is the gaussian hypergeometric function.

To insure the absolute convergence of the series (2.2), we must require $\operatorname{Re}(c-a-$ $\mu-1)>0$.

Consequently, taking into account that $[3, \mathrm{p} .50]$

$$
F(a, \mu+1 ; c ; 1)=\frac{\Gamma(c) \Gamma(c-a-\mu-1)}{\Gamma(c-a) \Gamma(c-\mu-1)},
$$

we obtain

$$
\begin{aligned}
J(a, \mu) & =\Gamma(\mu+1) \frac{\Gamma(c) \Gamma(c-a-\mu-1)}{\Gamma(c-a) \Gamma(c-\mu-1)} \\
c & \neq 0,-1,-2, \ldots, \mu>-1, \operatorname{Re}(c-a-\mu-1)>0 .
\end{aligned}
$$

We now consider the problem of evaluating the integrals (1.1).

We first examine the case $\rho=1$. Differentiating (2.3) with respect to $\mu$ gives

$$
\begin{aligned}
& \frac{\partial}{\partial \mu} J(a, \mu)= I_{1}(a, \mu) \\
&= \Gamma(\mu+1) \frac{\Gamma(c) \Gamma(c-a-\mu-1)}{\Gamma(c-a) \Gamma(c-\mu-1)} \\
& \quad \cdot\{\psi(\mu+1)+\psi(c-\mu-1)-\psi(c-a-\mu-1)\}, \\
& c \neq 0,-1,-2, \ldots, \mu>-1, \operatorname{Re}(c-a-\mu-1)>0,
\end{aligned}
$$


where $\psi(x)=\Gamma^{\prime}(x) / \Gamma(x)$ is the logarithmic derivative of the gamma function.

The evaluation of (1.1) when $\rho=2,3, \ldots$ can be obtained by repeatedly differentiating (2.4) with respect to $\mu$. We shall only examine, with some details, the case $\rho=2$.

By a partial differentiation of (2.4) with respect to $\mu$, we have

$$
\begin{aligned}
\frac{\partial^{2}}{\partial \mu^{2}} J(a, \mu)= & I_{2}(a, \mu) \\
= & \Gamma(\mu+1) \frac{\Gamma(c) \Gamma(c-a-\mu-1)}{\Gamma(c-a) \Gamma(c-\mu-1)} \\
& \cdot\left\{[\psi(\mu+1)+\psi(c-\mu-1)-\psi(c-a-\mu-1)]^{2}\right. \\
& \left.\quad+\left[\psi^{\prime}(\mu+1)+\psi^{\prime}(c-a-\mu-1)-\psi^{\prime}(c-\mu-1)\right]\right\}, \\
c \neq & 0,-1,-2, \ldots, \mu>-1, \operatorname{Re}(c-a-\mu-1)>0 .
\end{aligned}
$$

\section{Some sequences}

In this section we will evaluate the sequences of integrals (1.1) and (1.2) obtained from (2.3), (2.4), (2.5) when we change $a$ into $a-n$, where $n=0,1,2, \ldots$ and $a \neq 0$.

By remembering that $\Gamma(x+1)=x \Gamma(x)$, we may derive a useful algorithm for the computation of the moments $J(a-n, \mu)$.

Indeed, it is easily seen that, by putting

$$
J(a-n, \mu)=\alpha_{n}
$$

that is,

we have

$$
\alpha_{n}=\Gamma(\mu+1) \frac{\Gamma(c) \Gamma(c-a-\mu+n-1)}{\Gamma(c-a+n) \Gamma(c-\mu-1)}
$$

$$
\begin{aligned}
\alpha_{n+1} & =\alpha_{n} \frac{c-a-\mu+n-1}{c-a+n}, \quad n=0,1,2, \ldots, \\
\alpha_{0} & =J(a, \mu) .
\end{aligned}
$$

Moreover, for the computation of (2.4) when $a$ is substituted by $a-n, n=0,1,2, \ldots$, if we use the previously recalled property of function $\Gamma(x)$ and the recurrent relation $\psi(x+1)=\psi(x)+1 / x$, we may construct, in addition to (3.1), the following sequence

$$
\begin{aligned}
\beta_{n+1} & =\beta_{n}+\frac{1}{c-a-\mu+n-1}, n=0,1,2, \ldots, \\
\beta_{0} & =\psi(\mu+1)+\psi(c-\mu-1)-\psi(c-a-\mu-1),
\end{aligned}
$$

hence

$$
I_{1}(a-n, \mu)=\alpha_{n} \cdot \beta_{n}, n=0,1,2, \ldots
$$

Similarly, by replacing in (2.5) $a$ with $a-n, n=0,1,2, \ldots$, and recalling that $\psi^{\prime}(x+1)=\psi^{\prime}(x)-1 / x^{2}$, we obtain, together with the recurrent relationships (3.1) and 
(3.2), the recursion formula

$$
\begin{aligned}
\gamma_{n+1} & =\gamma_{n}-\frac{1}{(c-a-\mu+n-1)^{2}}, n=0,1,2, \ldots, \\
\gamma_{0} & =\psi^{\prime}(\mu+1)+\psi^{\prime}(c-a-\mu-1)-\psi^{\prime}(c-\mu-1),
\end{aligned}
$$

and, finally, the useful algorithm

$$
I_{2}(a-n, \mu)=\alpha_{n}\left\{\beta_{n}^{2}+\gamma_{n}\right\}, n=0,1,2, \ldots
$$

\section{Particular cases}

The results of the previous Sections reduce to the Gatteschi ones [2] when we assume $a=-n$ in (2.3), (2.4) and (2.5) or $a=0$ in Section 3 and, after setting $c=\alpha+1$, we change $\mu$ in $\alpha+\mu$.

This can be shown remembering that the Confluent Hpergeometric functions reduce to Laguerre polynomials when $a=-n, n=0,1,2, \ldots$, and, more precisely,

$$
L_{n}^{(\alpha)}(x)=\left(\begin{array}{c}
n+\alpha \\
n
\end{array}\right) \phi(-n, \alpha+1 ; x) .
$$

For instance from (2.3) we derive

$$
\int_{0}^{\infty} e^{-x} x^{\alpha+\mu} L_{n}^{(\alpha)}(x) d x=\frac{(-1)^{n}}{n !} \frac{\Gamma(\mu+1) \Gamma(\mu+\alpha+1)}{\Gamma(\mu-n+1)},
$$

which is the formula given by Gatteschi [2, p.1295].

We consider now the case $\mu+1-c=m, m=0,1,2, \ldots$ Recalling that, for any integer $r \geq 0$,

$$
\lim _{\epsilon \rightarrow 0} \frac{\psi(-r+\epsilon)}{\Gamma(-r+\epsilon)}=(-1)^{r-1} r !
$$

from (2.4) we obtain

$$
\begin{aligned}
I_{1}(a, \mu) & =\lim _{\epsilon \rightarrow 0} I_{1}(a, \mu-\epsilon) \\
& =\Gamma(\mu+1) \Gamma(c) \frac{\Gamma(c-a-\mu-1)}{\Gamma(c-a)} \lim _{\epsilon \rightarrow 0} \frac{\psi(c-\mu-1+\epsilon)}{\Gamma(c-\mu-1+\epsilon)} \\
& =(-1)^{m-1} \frac{\Gamma(c)}{\Gamma(c-a)} \Gamma(\mu+1) \Gamma(-a-m) \Gamma(m+1), \\
& \mu+1-c=m, m=0,1,2, \ldots, \operatorname{Re}(a)<-m, \mu>-1 .
\end{aligned}
$$

Analogously, for the evaluation of $I_{2}(a, \mu)$, we have from (2.5)

where

$$
\begin{aligned}
I_{2}(a, \mu) & =\lim _{\epsilon \rightarrow 0} I_{2}(a, \mu-\epsilon) \\
& =\frac{\Gamma(\mu+1) \Gamma(c) \Gamma(c-a-\mu-1)}{\Gamma(c-a)}\{A(\mu)+2 B(\mu)\},
\end{aligned}
$$




$$
\begin{aligned}
& A(\mu)=\lim _{\epsilon \rightarrow 0} \frac{\psi^{2}(c-\mu-1+\epsilon)-\psi^{\prime}(c-\mu-1+\epsilon)}{\Gamma(c-\mu-1+\epsilon)} \\
& B(\mu)=\lim _{\epsilon \rightarrow 0}\left\{[\psi(\mu+1-\epsilon)-\psi(c-a-\mu-1+\epsilon)] \frac{\psi(c-\mu-1+\epsilon)}{\Gamma(c-\mu-1+\epsilon)}\right\} .
\end{aligned}
$$

By means of the two series expansions

$$
\begin{array}{ll}
\Gamma(x)=\frac{(-1)^{r}}{r !} \frac{1}{x+r}+\sum_{k=0}^{\infty} a_{k}(x+r)^{k}, & r=0,1,2 \ldots, \\
\psi(x)=\frac{-1}{x+r}+\psi(1+r)+\sum_{k=1}^{\infty} b_{k}(x+r)^{k}, &
\end{array}
$$

which hold for $|x+r|<1$, it is easily seen that

$$
\begin{aligned}
& A(\mu)=(-1)^{\mu-c} 2 \psi(2+\mu-c) \Gamma(2+\mu-c), \\
& B(\mu)=(-1)^{\mu-c} \Gamma(2+\mu-c)\{\psi(\mu+1)-\psi(c-a-\mu-1)\} .
\end{aligned}
$$

Hence, substitution into (4.1), yields the final result

$$
\begin{aligned}
I_{2}(a, \mu) & =(-1)^{m-1} 2 \frac{\Gamma(\mu+1) \Gamma(c) \Gamma(-m-a)}{\Gamma(c-a)} \Gamma(m+1) \\
& \cdot\{\psi(m+1)+\psi(\mu+1)-\psi(-m-a)\}, \\
& +1-c=m, m=0,1,2, \ldots, \operatorname{Re}(a)\langle-m, \mu\rangle-1 .
\end{aligned}
$$

\section{References}

[1] J. L. Blue, "A Legendre polynomial integral," Math. Comp., 33(1979), 739-741.

[2] L. Gatteschi, "On Some Orthogonal Polynomial Integral," Math. Comp., 35(1980), 1291-1298.

[3] L. Gatteschi, "Funzioni Speciali," UTET, Torino(1973).

[4] W. Gautschi, "On the Construction of Gaussian Quadrature Rules from Modified Moments," Math. Comp., 24(1970), 245-260.

[5] W. Gautschi, On the preceding paper "A Legendre polynomial integral" by J. L. Blue, Math. Comp., 33(1979), 742-743.

[6] S. L. Kalla, S. Conde and Y. L. Luke, "Integrals of Jacobi Functions," Math. Comp., 38(1982), 207-214.

[7] S. L. Kalla and S. Conde, "Integrals of generalized Laguerre polynomials," Serdica, 9(1983), 230234.

Dipartimento di Matematica dell'Università, Via Carlo Alberto 10, I-10123 Torino, Italy. 